\title{
Advantage of four-electrode over two-electrode defibrillators
}

\author{
J. Bragard ${ }^{1}$, A. Šimić ${ }^{1}$, D. Laroze ${ }^{2,3}$, J. Elorza ${ }^{1}$ \\ 1 Physics 85 Applied Math. Dept., Navarra University, E-31080 Pamplona, Spain \\ 2 Instituto de Alta Investigacion, Universidad de Tarapaca, Casilla 7D, Arica, Chile \\ 3 SUPA School of Physics and Astronomy, University of Glasgow, Glasgow G12 8QQ, UK
}

(Dated: November 30, 2015)

\begin{abstract}
Defibrillation is the standard clinical treatment used to stop ventricular fibrillation. An electrical device delivers a controlled amount of electrical energy via a pair of electrodes in order to reestablish the normal heart rate. We propose a new technique that is a combination of biphasic shocks applied with a four-electrode system rather than the standard two-electrode system. We use a numerical model of a one-dimensional ring of cardiac tissue in order to test and evaluate the benefit of such a new technique. We compare three different shock protocols, namely, a monophasic and two types of biphasic shocks. The results obtained by using a four-electrode system are compared quantitatively with those obtained with the standard two-electrode system. We find that a huge reduction in defibrillation threshold is achieved with the four-electrode system. For the most efficient protocol (asymmetric biphasic), we obtain a reduction in excess of $80 \%$ in the energy required for a defibrillation success rate of $90 \%$. The mechanisms of successful defibrillation are also analyzed. This reveals that the advantage of asymmetric biphasic shocks with four electrodes lies in the duration of the cathodal and anodal phase of the shock.
\end{abstract}

\section{INTRODUCTION}

The electrical activity of a fibrillating heart is in a highly disorganized dynamical state maintained by one or more meandering spiral waves [1]. If not treated within minutes, ventricular fibrillation is lethal. The only existing medical treatment for ventricular fibrillation is defibrillation achieved by imposing electric shocks. This means the application of one or several external stimuli via two electrodes placed either externally over the chest or implanted subcutaneously in case of the internal cardioverter-defibrillator (ICD). The delivered energy during such shocks is between 150 to $360 \mathrm{~J}$ in the case of transthoracic defibrillation [2-4] and between 30 to 40 $\mathrm{J}$ in the case of ICD [5]. Unsurprisingly, in view of the large amount of energy involved in defibrillatory shocks, several side-effects have been reported, including pain [6], cardiac hemodynamics malfunctions [7], contractile malfunctions, etc. ICD devices have an additional size problem, as their size scales with the energy required. Nowadays, for both external and implanted defibrillators, the defibrillation protocol is biphasic: the polarity of the electrodes is reversed during the course of the defibrillation shock $[5,8]$. The first manufactured defibrillators, however, were monophasic. The technique of polarity reversal has allowed to achieve an energy saving of $25 \%$ for the external defibrillators [4]. Monophasic shocks delivered typically between 200 and $360 \mathrm{~J}$ of energy and the biphasic defibrillators deliver about 150 to $200 \mathrm{~J}[2,4,8,9]$. There have been different attempts to reduce the defibrillation thresholds by, e.g., reversing the polarity of the shock during the defibrillation and optimizing the reversal time, waveform and duration of the shock [5, 8, 9].

In this paper we study a new technique that is based on combining a four-electrode system with biphasic shocks rather than using the standard two-electrode system. In order to test the efficiency of this new technique, we use a numerical model to evaluate the benefits of such a fourelectrode system. The numerical model describes the dynamics of the action potential propagation in a onedimensional ring of cardiac tissue. The electrical behavior of the cardiac tissue is modeled through the standard bidomain model [10], and the Beeler-Reuter model [11] is used for the active properties of the membrane. We test three different shock types, viz., monophasic and two types of biphasic shocks. The results obtained with the new four-electrode technique are compared with those obtained with the standard two-electrode technique. We observe a drastic reduction in defibrillation threshold with the four-electrode technique. Quantitatively, by using the four-electrode system, a reduction of an order of magnitude in the energy needed for defibrillation is achieved. This huge reduction in energy opens up the perspective of building much more efficient defibrillator devices.

The present study is motivated by the analysis of results obtained with the standard two-electrode system in a simple model of a one-dimensional ring of cardiac tissue [12] recently developed by the authors. In this study [12], it was shown that the threshold $\mathrm{E}_{50}$, the applied electric field corresponding to a $50 \%$ of probability of success for defibrillation shocks, is achieved mainly by front to front interactions or interactions of the front with the refractory tissue, while the threshold E $_{90}$ needed for a $90 \%$ of probability of success is mainly obtained through a different mechanism, consisting of a direct activation of the whole cardiac tissue. Examining the mechanisms for defibrillation at low energy (i.e. small applied electric fields), led to the hypothesis that the addition of two electrodes might substantially reduce the values of $\mathrm{E}_{90}$. The present work confirms this hypothesis at least in a simple one-dimensional model of cardiac tissue.

The paper is organized as follows. In Section II, we describe the mathematical model that is used through- 


\section{University of Glasgow}

B ragard, J., ' imi •, A., L aroze, D., and Elorza, J. (2015) A dvantage of fourelectrode over two-electrode defibrillators. Physical Review E, 92, 062919.

There may be differences between this version and the published version. $Y$ ou are advised to consult the publisher's version if you wish to cite from it.

http://eprints.gla.ac.uk/113329/

Deposited on: 22 December 2015

Enlighten - R esearch publications by members of the U niversity of Glasgow http://eprints.gla.ac.uk 
out the paper to test the different defibrillation protocols and we detail the methods that are used to analyze the simulations. In Section III, the results of the numerical simulations of the four-electrode system are compared with those obtained with the two-electrode system. In addition, the mechanisms associated with successful defibrillation events are analyzed. In Section IV, we discuss the limitations of the model and possible extensions to more realistic models in two and three spatial dimensions. We also comment about the possible medical applications of our results. In Section $\mathrm{V}$ we draw some conclusions of our work.

\section{MODEL AND METHODS}

A reentrant wave in a one-dimensional system is often used as a simplified model of reentrant waves in higher dimensions. This simplification of tachyarrythmic behavior was used to study the interaction of reentrant dynamics with external stimuli. This simplification was exploited in a seminal work by Glass and Josephson [13] in the 1990s, in which a one dimensional geometry was used to study the annihilation and the resetting of reentrant waves. This original study was further broadened by the inclusion of conductivity heterogeneities and multiple stimuli [12, 14-18].

\section{A. Governing Equations}

The propagation of an electric wave (action potential) in the cardiac tissue is described using the standard bidomain model. This represents the myocardium as a continuum where the cell conductances are homogenized in space [10]. The transmembrane potential $V_{m}=\Phi_{i}-\Phi_{e}$ satisfies

$$
\begin{gathered}
\frac{\partial V_{m}}{\partial t}=-\frac{i_{B R}+i_{e p}+i_{f u}}{C_{m}}+\nabla \cdot\left(\mathbf{D}_{\mathbf{i}} \cdot \nabla V_{m}\right)+\nabla \cdot\left(\mathbf{D}_{\mathbf{i}} \cdot \nabla \Phi_{e}\right), \\
\nabla \cdot\left[\left(\mathbf{D}_{\mathbf{i}}+\mathbf{D}_{\mathbf{e}}\right) \cdot \nabla \Phi_{e}\right]=-\nabla \cdot\left(\mathbf{D}_{\mathbf{i}} \cdot \nabla V_{m}\right)-\frac{i_{e}}{\chi C_{m}},
\end{gathered}
$$

where $\Phi_{e}$ is the extracellular electrical potential, $\Phi_{i}$ is the intracellular electrical potential, $C_{m}$ is the membrane capacitance $\left(\approx 1 \mu \mathrm{F} / \mathrm{cm}^{2}\right.$ for the membrane of the cardiac myocytes), $\chi$ is the myocyte surface-to-volume ratio $\left(\approx 1,400 \mathrm{~cm}^{-1}\right)$ and $\mathbf{D}_{\mathbf{i}}$ and $\mathbf{D}_{\mathbf{e}}$ denote the intracellular and extracellular diffusion tensors. These tensors are related to the conductivity tensors $\sigma$ through the simple relation $\mathbf{D}_{\mathbf{i}, \mathbf{e}}=\sigma_{\mathbf{i}, \mathbf{e}} /\left(\chi C_{m}\right)$. In Eq. (1), $i_{m}=i_{B R}+i_{e p}+i_{f u}$ denotes the total transmembrane current which is model-specific (see Sec. II B for details) and $i_{e}$ denotes the injected current in the extracellular region. This last term in Eq. (1) makes it possible to use the bidomain model in order to simulate applied stimuli to the cardiac tissue at the electrode locations.
Resistive discontinuities in the heart tissue, such as cell-to-cell gap junctions, intracellular clefts or fiber orientations, act as redistribution centers of intracellular and extracellular currents and locally hyperpolarize or depolarize the tissue [19]. If the depolarization is strong enough at the heterogeneity location, an excitation wave can be produced [20]. These local hyperpolarizations or depolarizations are known as virtual electrodes (VE) [21]. In the present model, in order to mimic the heterogeneity of the cardiac tissue, we have superimposed to the intracellular conductance a Gaussian noise in such a way that the intracellular diffusion tensor is modified according to

$$
D_{i}\left(x_{i}\right)=\bar{D}_{i}\left(1+\tilde{s} \delta_{i}\right),
$$

where $\bar{D}_{i}$ is the average value of intracellular diffusion, set to $1.5 \cdot 10^{-3} \mathrm{~cm}^{2} / \mathrm{ms}, \delta_{i}$ is a random variable drawn from a Gaussian distribution with zero mean and unit variance and $\tilde{s}$ is a parameter that controls the strength of the heterogeneities and is set to $\tilde{s}=0.15$. Note that $x_{i}$ denotes the grid location and that the grid spacing $\Delta_{x}=$ $x_{i+1}-x_{i}$ has been set in this study to $\Delta_{x}=0.025 \mathrm{~cm}$. Here, the extracellular diffusion is taken constant as $D_{e}=$ $1.5 \cdot 10^{-3} \mathrm{~cm}^{2} / \mathrm{ms}$. A more realistic description of the tissue heterogeneities should also include heterogeneities in the extracellular region as well as a model for the gap junction dynamics through the connexins [22] but this is left for a future work.

\section{B. Membrane Model}

we use a modified version of the Beeler-Reuter (BR) [11] model to describe the active properties of the cell membrane. The original BR model contains four contributions in the transmembrane current: $i_{B R}=i_{K}+i_{x}+$ $i_{N a}+i_{s}$. The ionic current $i_{B R}$ is the sum of currents carried by the potassium ions $\left(i_{K}\right.$ is the time-independent outward current, $i_{x}$ is the time-dependent outward current), the sodium ions ( $i_{N a}$ is the fast excitatory inward current), and the calcium ions $\left(i_{s}\right.$ is the slow inward current). We take the same equations and parameter values as in Ref. [23]. In particular, we use $\sigma=0.7$ for the reduction of the time constants associated with the calcium activation and inactivation gates $d, f$, respectively. Furthermore, the calcium conductance is slightly modified and set to $g_{s}=0.07 \mathrm{mS} / \mathrm{cm}^{2}$. This choice of parameter values favors the instability of the action potential wave and places the system in a state of cardiac alternans (see details below and Fig.1a).

The parameters of the BR model were originally obtained by fitting experiments performed in the normal physiological range of transmembrane potentials. Some modifications are needed to the original BR model in order to describe the phenomena occurring at very low or very high transmembrane potentials. These situations are commonly encountered when strong extracellular stimuli are applied as it is the case during defibrillation shocks. 
It is known from electrophysiology experiments [24] that anodal stimulation, although causing the local hyperpolarization of the underlaying tissue, can elicit a propagating front upon termination of the stimulus. This effect is called "anode break excitation" (ABE) [25]. Although this effect has been observed in experiments [2628 ] on canine, rat and guinea pig ventricular myocytes, it is not captured by the equations of the standard BR model. Here, to account for the ABE, we adopt the model developed by Ranjan et al. [28]. In the latter model, $\mathrm{ABE}$ is brought by the hyperpolarization induced current $i_{f u}$ in combination with time-dependent blockage and unblockage of the potassium current. It is this timedependent unblocking of the outward potassium current that potentiates the effect of the inward "funny current" and brings the transmembrane potential over the threshold value for the elicitation of a new action potential. We adopt a full description of the Ranjan model of the $i_{f u}$ current as well as the addition of a new gate variable to transform the time-independent current $i_{K}$ into a time-dependent current [28]:

$$
i_{f u}=g_{f u} f u\left(V-E_{f u}\right),
$$

where $E_{f u}=-29 \mathrm{mV}$ is the reversal potential for $i_{f u}$, $g_{f u}=0.1 \mathrm{mS} / \mathrm{cm}^{2}$ is the conductance associated with the "funny current" and $f u$ is a new gate variable based on a Hodgkin-Huxley-type dynamics [28].

A second important modification brought to the standard BR model concerns the electroporation phenomenon. This phenomenon consists in the opening of reversible, water-filled pores as a response to very high applied electric fields. Experiments have shown that the transmembrane potential saturates with increasing applied electric fields [29]. This behavior was not captured with the first available mathematical physiological models of the membrane kinetics. De Bruin and Krassowska [30] suggested that this behavior can be attributed to the electroporation phenomenon and they developed a mathematical model in which electroporation current $\left(i_{e p}\right)$ is taken into account [30]:

$$
\begin{gathered}
i_{e p}=g_{p}\left(V_{m}\right) N V_{m} \\
\frac{d N}{d t}=\alpha \exp \left(\beta V_{m}^{2}\right)\left[1-\frac{N}{N_{0}} \exp \left(-q \beta V_{m}^{2}\right)\right]
\end{gathered}
$$

where the conductance $g_{p}\left(V_{m}\right)$ is modeled as an instantaneous function of the transmembrane potential [30] and $N$ is the membrane pore density. Eq.(5) describes the first-order kinetics of the pore density. The parameter values $\alpha=200 \mathrm{~cm}^{-2} \mathrm{~ms}^{-1} ; \beta=6.2510^{-5} \mathrm{mV}^{-2}$ and $N_{0}=1.510^{5} \mathrm{~cm}^{-2}$ used here are the same as in the original paper by De Bruin and Krassowska [30]. The strength of this electroporation current depends on the opening and resealing of pores and has a nonlinear dependence on $V_{m}$ as shown in Eq.(5). The electroporation current has been incorporated to our transmembrane model in
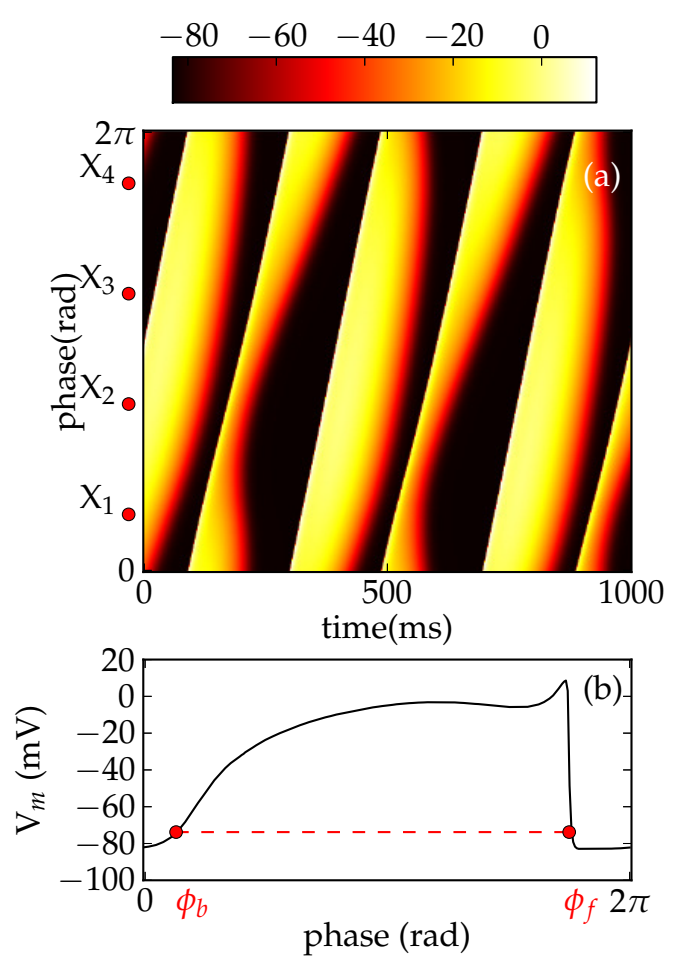

FIG. 1: (Color online) a) Space-time plot of the discordant-alternans wave on the one-dimensional ring. The vertical axis is space $(L=6.7 \mathrm{~cm})$ and it is rescaled to the phase variable varying in $[0,2 \pi]$ radians. The color-coding quantifies the values of the transmembrane potential $V_{m}$ in $\mathrm{mV}$ units (Top color bar). The locations of the four electrodes $X_{i}(\mathrm{i}=1, \ldots, 4)$ are also depicted. $\left.\mathrm{b}\right)$

Snapshot of $\mathrm{V}_{m}$ taken from (a) at time $t=400 \mathrm{~ms}$.

order to avoid the transmembrane potential reaching unphysically large values (positive and negative) during the shocks. In our simulations, the $i_{e p}$ current is included to Eq.(1) only if $V_{m}<-180 \mathrm{mV}$ or $V_{m}>150 \mathrm{mV}$, i.e. only when strong deviations from the physiological conditions are observed.

\section{Numerical Defibrillation Experiments}

All the calculations presented in this paper have been performed on a one-dimensional ring of cardiac tissue. The ring size $(L=6.7 \mathrm{~cm})$ was selected in order to get a sustained discordant-alternans dynamics. This dynamical state is of interest because it is known to be a precursor of the fibrillation state [31]. A typical space-time plot of the wave propagation, depicted in Fig.1a, shows the periodic propagation of the action potential along the ring and the irregular variation of the action potential duration. Also shown in Fig.1a are the locations of the four actuating electrodes indicated by the labels $X_{i}$ $(i=1, \ldots, 4)$ and corresponding dots. The four electrodes are equispaced along the circumference of the ring. The 
a)

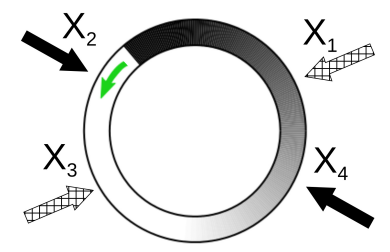

b)

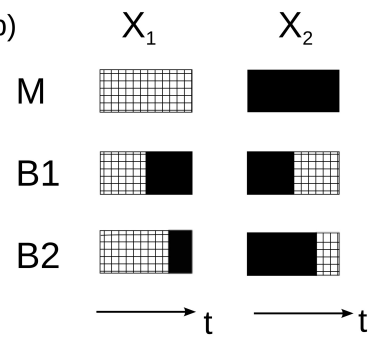

FIG. 2: Illustration of the four-electrode system setup.

Hatched arrows and hatched rectangles denote the anodes (injection of positive charges into the extracellular region). Black arrow and black rectangles denote the cathodes. a) Positions of the electrodes on the ring (stimulus sites) for the monophasic and first phase of the biphasic shocks. b) Illustration of the applied monophasic, biphasic I and biphasic II shocks through the electrodes $\mathrm{X}_{1}$ and $\mathrm{X}_{2}$. The electrode $\mathrm{X}_{3}$ receives the same charge as the electrode $\mathrm{X}_{1}$. Electrode $\mathrm{X}_{4}$ receives the same charge as the electrode $\mathrm{X}_{2}$. The total shock duration is always set to $8 \mathrm{~ms}$. Note that the waveforms are rectangular.

discordant-alternans dynamics shown in Fig.1a is characterized by two periods, one associated with the propagation of the wave along the ring and one associated with the intrinsic action potential duration variation. The two frequencies associated with this quasi-periodic dynamics can be easily determined by computing the Fourier spectrum of the signal at a fixed location. With the parameters of our model, the two frequencies are approximately $f_{1}=5.07 \mathrm{~Hz}$ and $f_{2}=0.33 \mathrm{~Hz}$. The first frequency is associated with the time taken by a wave to go around the ring, $T_{1}=197 \mathrm{~ms}$. The second frequency is associated with the time taken by a node (location where the action potential has a minimum duration) to go around the ring, $T_{2}=3,030 \mathrm{~ms}$. Figure $1 \mathrm{~b}$ displays a snapshot of an action potential at time $t=400 \mathrm{~ms}$. The positions of the wave front $\phi_{f}$ and the wave back $\phi_{b}$ are also indicated. Note that in the remainder of the paper, the space variable $x \in[0, L]$ along the circumference of the ring is converted into a phase variable $\phi \in[0,2 \pi]$. Following standard practice, the front locations are calculated from a $90 \%$ decrease of the maximum depolarization value of $\mathrm{V}_{m}\left(A P D_{90}=\left(\phi_{f}-\phi_{b}\right) \bmod 2 \pi\right)$. The numerical experiments are designed to test defibrillation shocks in the following way. From the undisturbed quasiperiodic dynamics (see Fig.1a), we pick up as initial conditions for defibrillation a very large sample $(n=2,000)$ of states. The time interval between consecutive saved initial states is randomly chosen in the range of $[28,38] \mathrm{ms}$. We have verified that for such time the cross-correlation between successive states has already decreased substantially. Specifically, the time correlation function computed from the dynamics shown in Fig.1a is in the range between $[0.42,0.2]$ if time is in the range of $[28,38] \mathrm{ms}$. This ensures some degree of statistical independence be-

tween the saved initial states. Then for all the elements of this large sample, a shock of $8 \mathrm{~ms}$ duration is applied through the point electrodes as illustrated in Fig.2. Three different shock protocols have been tested as indicated in Fig.2. Monophasic (M), in which the polarity of the electrodes is maintained during the whole shock duration, symmetric biphasic in which the electrode polarity is reversed at the middle of the shock and asymmetric biphasic in which the polarity is reversed at $75 \%$ of the total shock duration. We will refer here to symmetric biphasic as biphasic 1 (B1) and to asymmetric biphasic as biphasic 2 (B2). The defibrillation is classified as successful if all the electrical activity has stopped in the system 1,000 ms after the shock. For the two-electrode system, the electrodes are located $\pi$ radians apart on the ring and for the four-electrode system (FE) they are located at a distance of $\pi / 2$ radians apart (see Fig.2). Note that the waveforms for the protocols are rectangular and are an approximation of the exponentially decaying waveforms of commercial defibrillators.

The percentage of success in removing all the electrical activity in the ring is calculated by averaging the results obtained with all different initial conditions $(n=2,000)$ and for all the different noise distributions of tissue heterogeneities. The tissue heterogeneities (see Sec. II A) are modeled by 50 independent Gaussian noise distributions added to the internal conductance in the case of the four-electrode system and 80 independent Gaussian noise distributions in the case of the two-electrode system [12]. These simulations are repeated for increasing values of the applied electric field $E(\mathrm{~V} / \mathrm{cm})$. The latter is measured by $E=\left(\Phi_{a}-\Phi_{c}\right) / \Delta L$, where $\Phi_{a}$ and $\Phi_{c}$ denote the extracellular electrical potential of consecutive anode and cathode, respectively and $\Delta L=$ the separation between them, here $\Delta L=6.7 \mathrm{~cm} / 4=1.675 \mathrm{~cm}$ for the four-electrode system and $\Delta L=6.7 \mathrm{~cm} / 2=3.35 \mathrm{~cm}$ for the two-electrode system. In the present configurations the electric field is constant in magnitude but switches direction after each electrode.

\section{Numerical Techniques}

The parabolic equation associated to the transmembrane potential $V_{m}$ and the ODEs associated with the gate dynamics were solved using a simple forward Euler method. The Poisson equation, the most time consuming part of the computation, was solved using the KSP solver (KSPSolve) of the PETSc library [32]. The integration method is based on the generalized minimal residual method (GMRES) [33]. During the shock application and for $10 \mathrm{~ms}$ after the shock termination, the time step is fixed to $\delta t=0.001 \mathrm{~ms}$, while for the rest of the simulation the time step was set to $\delta t=0.01 \mathrm{~ms}$. In order to speed up the calculations, lookup tables were created and used whenever complex functions were repeatedly evaluated as for example in the gate variable evolution equations. Periodic boundary conditions were 


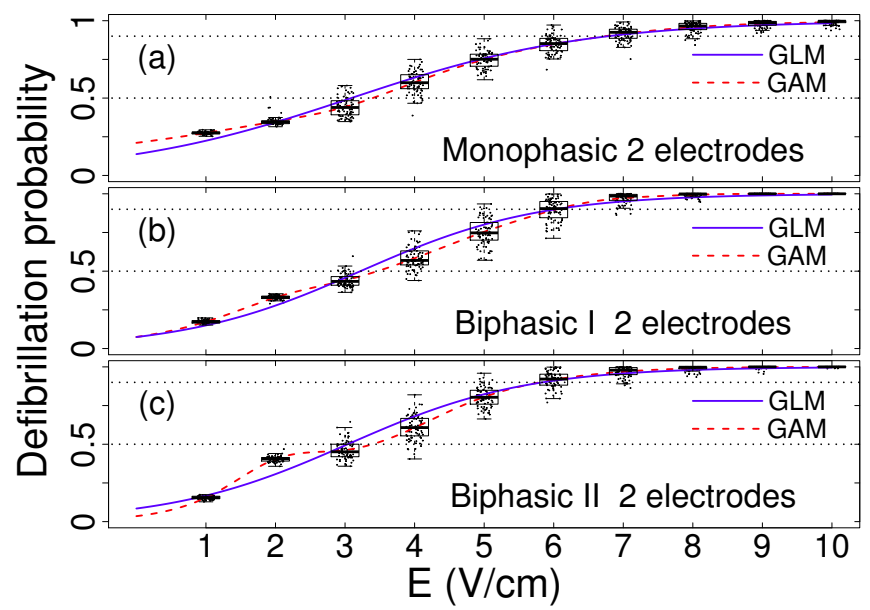

FIG. 3: (Color online) Two-electrode system.

Numerical data (boxplot) and fitted dose-response curve (according to Eq.(6) solid blue lines; and Eq.(7) dashed red lines) for monophasic (a), biphasic I (b) and biphasic II (c) protocols.

used to simulate the one dimensional ring geometry. It means that at each time step we impose $V_{m}(0)=V_{m}(N)$ and $V_{m}(N+1)=V_{m}(1)$ for the grid points $i=0$ and $i=N+1$ and the same periodic boundary conditions for the extracellular electrical potential $\Phi_{e}$. The spatial discretization mesh was fixed to $\delta x=0.025 \mathrm{~cm}$ and the total length of the ring was selected as $L=6.7 \mathrm{~cm}(\mathrm{~N}=268)$. The external stimulus is applied through current injection into the extracellular region using the $i_{e}$ term of the Eq.(1). The current injection sites or electrodes are placed equidistantly along the circumference of the ring as shown in Fig.2.

\section{E. Statistical Data Analysis}

\section{Dose-response curve}

Let us recall that the main purpose of the paper is to measure the efficacy of the defibrillation shocks when the applied electric field is increased. The dose-response curve which is a plot of the percentage of success versus the applied electric field is commonly used in assessing defibrillation efficacy. The data typically show a sigmoidal function that tends to zero for low applied fields and saturating to $100 \%$ success rate for high applied fields. The data are fitted with a logistic curve. This procedure was used for the data obtained by the authors in the case of the two-electrode system [12] (see Fig. 3).

The form of the logistic curve is given by :

$$
\log \left(\frac{p}{1-p}\right)=\beta_{0}+\beta_{1} E,
$$

where $p$ is the probability of success and $E$ is the applied electric field $(\mathrm{V} / \mathrm{cm})$ in the shock. The goodness of fit (GOF) for logistic functions can be assessed by computing the pseudo- $R^{2}$ given by McFadden [34] which is a generalization of the standard $R^{2}$ used in linear regression.

When we analyze the data for the four-electrode configuration (see Sec. III, Fig. 4) we will observe that the simple logistic curve Eq.(6) is not adequate to fit the data. In order to account for the added complexity in the data, we have used a generalized additive model (GAM) [35]. This model is a generalization of Eq.(6) that takes the following form:

$$
\log \left(\frac{p}{1-p}\right)=\beta_{0}+\beta_{1} \cdot s(E)
$$

in which $s(E)$ is a now a smooth function of the predictor $(E)$ that in our case is a linear combination of cubic splines that are determined to minimize the error of the fit through an optimization algorithm [35]. Here, the GAM fitting was performed by using the corresponding $\mathrm{R}$ package [36] mgcv [35, 37, 38]. The number of base functions (splines) used for the description of the function $s(E)$ was taken to be $(k=21)$ for the four-electrode fittings and $(k=10)$ for the two-electrode fittings. The improvement of the GAM fitting (Eq. (7)) over the classical logistic regression (Eq. (6)) is quantified by computing the corresponding pseudo- $R^{2}$ given by McFadden [34].

\section{Estimation of the standard errors by bootstrap techniques}

When using a fitting software one generally obtains the fitting curve plus the standard error (or confidence interval) for the fitting curve. The latter is computed by making a series of assumption on the statistical distribution of the data around the fitting curve. In the specific case of the generalized linear model (GLM) fitting one generally assumes that the deviance residuals follow a binomial or normal distribution [35]. For the data that we have treated in this paper, for both cases (i.e. the two and the four-electrode system), the data do not follow such distribution. The easy way to evaluate standard errors of the fit parameters when the data do not follow the assumed distribution is to use bootstrap techniques [39]. The technique is based on the many repetitions of the data by resampling the available data set. In our case, it means that we make 10,000 copies of the data by extracting new data using resampling with allowed repetitions. Each one of the 10,000 copies are then fitted using GAM and GLM methods and the corresponding fitting parameter are extracted. Here, we are interested in extracting from the fitting curves the values $E_{50}$ and $E_{90}$ that correspond to the electric field associated with a $50 \%$ and $90 \%$ probability of defibrillation, respectively. The statistical distribution of the $E_{50}$ and $E_{90}$ extracted from the bootstrap follows (as expected) a normal distribution and the standard error is identified to the stan- 


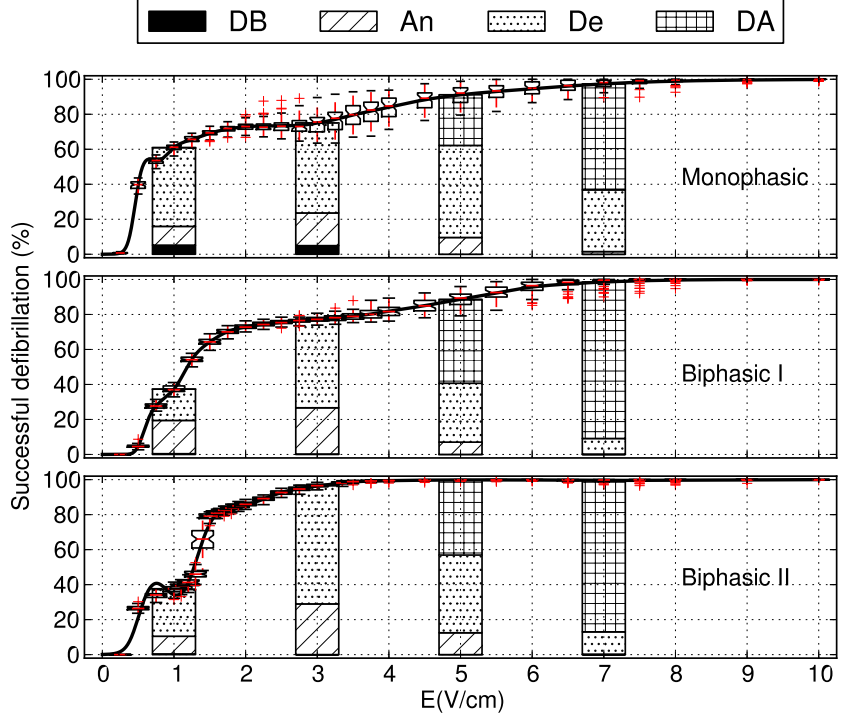

FIG. 4: Four-electrode system. Numerical data (boxplot) and fitted dose-response curve (according to Eq.(7)) for monophasic (top), biphasic I (middle) and biphasic II (bottom) protocol. Stacked bars represent

the classification of defibrillation mechanisms for electric fields corresponding to: $E=1 \mathrm{~V} / \mathrm{cm}, 3 \mathrm{~V} / \mathrm{cm}$, $5 \mathrm{~V} / \mathrm{cm}$ and $7 \mathrm{~V} / \mathrm{cm}$. Here, the abbreviations stand for : DB-direct block, An-annihilation, De-delayed block and DA-direct activation.

TABLE I: $\mathrm{E}_{90}$ and $\mathrm{E}_{50}$ estimated values plus standard error for the two-electrode and four-electrode systems using GAM fitting Eq.(7). pseudo- $R^{2}$ are also provided.

\begin{tabular}{|c|c|c|c|c|c|c|}
\hline \multirow[b]{2}{*}{ DP } & \multicolumn{3}{|c|}{4 electrodes } & \multicolumn{3}{|c|}{2 electrodes } \\
\hline & $\mathrm{R}^{2}$ & $E_{50}(V / \mathrm{cm})$ & $E_{90}(V / c m)$ & $\mathrm{R}^{2}$ & $E_{50}(\mathrm{~V} / \mathrm{cm})$ & $E_{90}(V / c m)$ \\
\hline M & 0.95 & $0.59 \pm 0.003$ & $4.75 \pm 0.15$ & 0.95 & $3.40 \pm 0.05$ & $6.74 \pm 0.09$ \\
\hline B1 & 0.98 & $1.21 \pm 0.005$ & $5.19 \pm 0.08$ & 0.96 & $3.47 \pm 0.04$ & $6.04 \pm 0.06$ \\
\hline B2 & 0.99 & $1.28 \pm 0.004$ & $2.32 \pm 0.01$ & 0.96 & $3.39 \pm 0.06$ & $5.80 \pm 0.07$ \\
\hline
\end{tabular}

dard deviation of the parameter estimates. The results obtained through the bootstrap techniques are gathered in Tables I \& II in Sec. III.

\section{RESULTS}

\section{A. Dose-response curve}

Figure 4 shows the numerical data and fitting results (according to Eq.(7)) corresponding to the four-electrode system and the three tested protocols. The curves for the monophasic and the biphasic I protocols in Fig. 4 display two plateaus: a) the expected saturation plateau for high field values and a second small plateau in the range of 2 to $3 \mathrm{~V} / \mathrm{cm}$. Therefore, the Eq.(6) for one-
TABLE II: $\mathrm{E}_{90}$ and $\mathrm{E}_{50}$ estimated values plus standard error for the two-electrode and four-electrode systems using GLM fitting Eq.(6). pseudo- $R^{2}$ are also provided.

\begin{tabular}{|c|c|c|c|c|c|c|}
\hline \multirow[b]{2}{*}{$\mathrm{DP}$} & \multicolumn{3}{|c|}{4 electrodes } & \multicolumn{3}{|c|}{2 electrodes } \\
\hline & $\mathrm{R}^{2}$ & $E_{50}(V / \mathrm{cm})$ & $E_{90}(V / c m)$ & $\mathrm{R}^{2}$ & $E_{50}(V / \mathrm{cm})$ & $E_{90}(V / \mathrm{cm})$ \\
\hline $\mathrm{M}$ & 0.78 & $0.97 \pm 0.03$ & $4.53 \pm 0.11$ & 0.94 & $3.09 \pm 0.03$ & $6.79 \pm 0.08$ \\
\hline B1 & 0.83 & $1.61 \pm 0.01$ & $4.31 \pm 0.06$ & 0.94 & $3.22 \pm 0.02$ & $6.03 \pm 0.06$ \\
\hline $\mathrm{B} 2$ & 0.94 & $1.17 \pm 0.01$ & $2.23 \pm 0.02$ & 0.94 & $3.04 \pm 0.02$ & $5.84 \pm 0.06$ \\
\hline
\end{tabular}

predictor logistic regression previously employed in the two-electrode system (see Fig.3) is no longer a suitable choice for fitting the data of the four-electrode system shown in Fig. 4 and the generalized additive model [35] described above needs to be used.

Tables I \& II gather all the information of the $E_{90}$ and $E_{50}$ threshold values extracted from the curve fittings using the GAM and GLM techniques, respectively. For the sake of comparison, we have provided the results of the $E_{90}$ and $E_{50}$ for the two types of fitting and also the pseudo- $R^{2}$ given by McFadden [34]. The standard errors were computed by bootstrap techniques (with 10,000 repetitions). The first observation is that for the data of the two-electrode system [12] both types of fitting give satisfactory results and especially for $E_{90}$ values we do not observe large differences between the two types of fitting. This no longer holds true for the four-electrode system where the values for the GLM and GAM fitting are discrepant. The GAM fittings provide much higher values for the pseudo- $R^{2}$ and therefore are a better model for our data. In the following we will analyze in more detail the values given in Table I.

The analysis of the threshold values $E_{90}$ are of high interest for the defibrillators. The difference in $E_{90}$ between the $\mathrm{M}$ and B2 protocol for the two-electrode system leads to a decrease in energy of approximately $26 \%$ in favor of the B2 protocol. This result follows very closely the values found in the medical literature [40,41]. The same comparison, in the case of the four-electrode system, leads to a decrease in energy of approximately $76 \%$ in favor of the B2 protocol when compared to the M protocol. Another important comparison is the difference between the $E_{90}$ values for the two- and four-electrode systems. The decrease in energies, according to the values given in Table I, between the four- and two-electrode system are : $50 \%$ for the M protocol; $26 \%$ for the B1 protocol and $84 \%$ for the B2 protocol.

\section{B. Defibrillation mechanisms}

A direct observation of the successful defibrillation events indicates that, as in the case of the two-electrode system, there are four distinct mechanisms: (1) Direct block (DB), (2) Annihilation (An), (3) Delayed block (De) and (4) Direct activation (DA). The four mecha- 
TABLE III: Classification of the shock outcomes obtained by the ANN analysis at four shock strengths $(E=1,3,5$ and $7 \mathrm{~V} / \mathrm{cm}$ ). The probability (in percents) and its standard deviation (in parentheses) are given for each possible shock outcome.

\begin{tabular}{|c|c|c|c|c|c|c|}
\hline $\mathrm{E}(\mathrm{V} / \mathrm{cm})$ & Protocol & Failure & Direct block & Annihilation & Delayed block & Direct activation \\
\hline \multirow{3}{*}{1} & Monophasic & 39.08 & $5.20_{(0.97)}$ & $10.73_{(0.28)}$ & 44.99 (1.04) & $0_{(-)}$ \\
\hline & Biphasic 1 & 62.69 & $0.42_{(0.59)}$ & $19.03(0.90)$ & 17.86 & $0_{(-)}$ \\
\hline & Biphasic 2 & 62.72 & $0.41(0.25)$ & $10.10_{(0.33)}$ & $26.78(0.41)$ & $0_{(-)}$ \\
\hline \multirow{3}{*}{3} & Monophasic & 25.01 & $4.92(0.31)$ & $18.68(0.57)$ & $51.39(0.56)$ & $0_{(-)}$ \\
\hline & Biphasic 1 & 22.69 & $0.25_{(0.14)}$ & 26.42 (1.10) & $50.65_{(1.10)}$ & $0_{(-)}$ \\
\hline & Biphasic 2 & 3.39 & $0.013_{(0.015)}$ & $28.97_{(1.25)}$ & $67.62_{(1.26)}$ & $0_{(-)}$ \\
\hline \multirow{3}{*}{5} & Monophasic & 8.85 & $0_{(-)}$ & $9.58_{(0.49)}$ & $52.61(0.75)$ & $28.95(0.80)$ \\
\hline & Biphasic 1 & 11.34 & $0_{(-)}$ & $7.03_{(0.74)}$ & $33.66_{(0.90)}$ & $47.97_{(0.76)}$ \\
\hline & Biphasic 2 & 0.19 & $0_{(-)}$ & $12.49_{(0.67)}$ & $44.41_{(0.91)}$ & $42.92_{(1.33)}$ \\
\hline \multirow{3}{*}{7} & Monophasic & 2.64 & $0_{(-)}$ & $1.51_{(0.41)}$ & $35.30(0.96)$ & $60.56(0.68)$ \\
\hline & Biphasic 1 & 1.44 & $0_{(-)}$ & $0.19_{(0.067)}$ & $8.93_{(1.10)}$ & $89.44_{(1.10)}$ \\
\hline & Biphasic 2 & 0.46 & $0_{(-)}$ & $0.25(0.12)$ & $12.77_{(1.79)}$ & $86.51_{(1.84)}$ \\
\hline
\end{tabular}

nisms typically occur at increasing values of the electric field $E$. Some examples are displayed in Fig. 5. The DB mechanism is specific of the monophasic protocol. In this case, the initial front is suddenly halted by a hyperpolarized region created by the anodal stimulus and the wave is directly blocked. If no other fronts are created by means of virtual or real electrodes, the defibrillation is successful. An illustration of the DB mechanisms is shown in Fig.5a where the shock strength is $E=1 \mathrm{~V} / \mathrm{cm}$ and the shock protocol is monophasic. A second type of successful defibrillation can be achieved by means of the annihilation (An) mechanism, in which all the electrical activity on the ring is stopped by the collision of two counter propagating fronts. Figure 5 b shows an example of the annihilation mechanism where the shock energy is $E=3 \mathrm{~V} / \mathrm{cm}$ and the shock protocol is the biphasic 2 . In this case, the electrode located at position $X_{3}$ is anodal in the first phase (from $t=0$ to $6 \mathrm{~ms}$ ) and cathodal in the second phase (from $t=6$ to $8 \mathrm{~ms}$ ). For the electrode at $X_{3}$, the short depolarization is sufficient for eliciting two new fronts. The downward front annihilates with the initial front that existed in the ring prior to the shock, and the upward front annihilates with the front elicited by the electrode located at position $X_{4}$. A third mechanism of defibrillation is the so-called delayed block (De). It consists in the blockage of the surviving front passing through a region with refractory tissue. An example of this mechanism is shown in Fig.5c. In this case, the shock energy is $5 \mathrm{~V} / \mathrm{cm}$ and the shock protocol is monophasic. In Fig.5c, one observes that the shock energy is sufficient to produce virtual electrodes (VE) and one of those VE produces a new front. This front propagates until it encounters a region of refractory tissue where it is blocked. Finally, the last mechanism is the so-called direct activation mechanism because the shock activates a large portion of the cardiac tissue. When the tissue returns back to the rest state, none of the waves survive. An
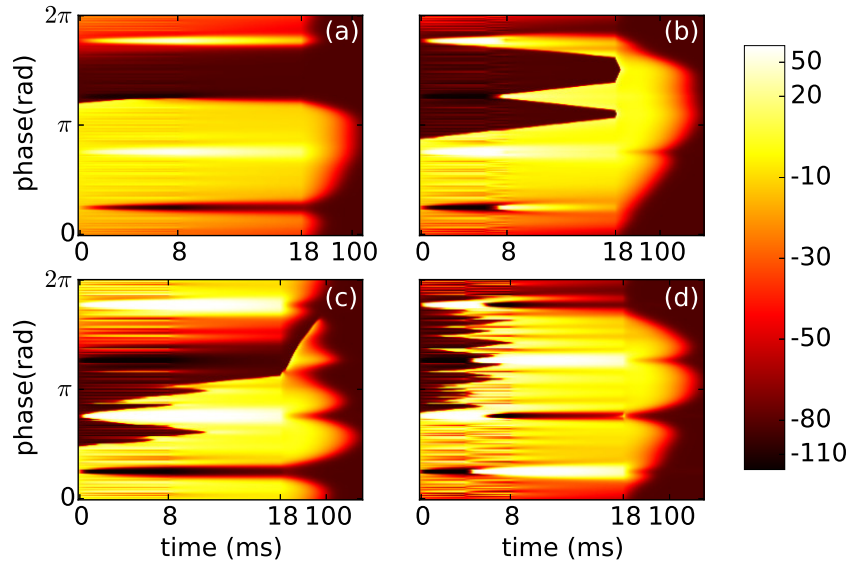

FIG. 5: Space-time plots of $V_{m}$ showing examples of defibrillation mechanisms. The shock is applied through a four-electrode system. (a) Direct block (Monophasic, $\mathrm{E}=1 \mathrm{~V} / \mathrm{cm}$ ) (b) Annihilation (Biphasic 2, $\mathrm{E}=3 \mathrm{~V} / \mathrm{cm}$ ) (c)

Delayed block (Monophasic, $\mathrm{E}=5 \mathrm{~V} / \mathrm{cm}$ ) (d) Direct

activation (Biphasic 1, E=7V/cm). The shock is applied during the first $8 \mathrm{~ms}$. The time scale of all the plots is magnified 10 times for $\mathrm{t} \in(0,18)$ with respect to $\mathrm{t}>18 \mathrm{~ms}$. A nonlinear color scale for $V_{m}$ is used for all the plots.

example of the direct activation mechanism is shown in Fig.5d. In this case the shock energy is equal to $7 \mathrm{~V} / \mathrm{cm}$ and the shock protocol is the biphasic 1 . The initial front is close to the $X_{2}$ electrode. The energy of the shock is large enough to produce many virtual electrodes in the region between the $X_{2}$ and $X_{4}$ electrodes.

Because of the large number of simulations, we have performed an automatic classification of the defibrillation mechanisms using artificial neural networks (ANN) [42]. 

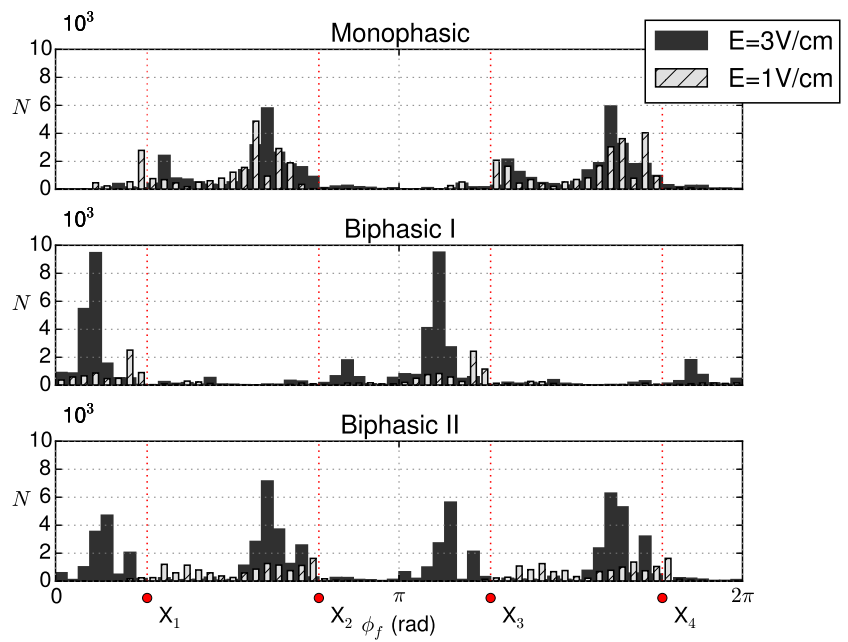

FIG. 6: Histograms showing the number of successful defibrillation trials via the delayed block mechanism. Here $\phi_{f}$ denotes the position where the blocked front was last detected before being blocked. Histograms are compared for monophasic (upper plot), biphasic 1 (middle plot) and biphasic 2 (lower plot) protocols. Note that the results for $E=1 \mathrm{~V} / \mathrm{cm}$ are depicted with narrower hatched bars for the sake of clarity.

The details of the classification method are described in the Appendix. Four values of the electric fields, i.e., $E=1$, 3,5 and $7 \mathrm{~V} / \mathrm{cm}$, were analyzed in detail for each protocol. The results are shown in Fig. 4 and summarized in Table III. For low shock energies, around $E=1 \mathrm{~V} / \mathrm{cm}$, the monophasic shock protocol is the more efficient and the two biphasic protocols have similar failure rate. Mean failure rates corresponding to the $\mathrm{M}, \mathrm{B} 1$ and $\mathrm{B} 2$ protocols are approximately $39 \%, 62.7 \%$ and $62.7 \%$, respectively. At low shock energy, we do not observe the creation of virtual electrodes, but some new fronts are created at the location of the physical electrodes, if the shock is depolarizing there. Figure 4 and Table III confirm that the better efficiency of monophasic shock at $\mathrm{E}=1 \mathrm{~V} / \mathrm{cm}$ is essentially due to the delayed block mechanism. Recall that the mean failure rates at the same energy $(\mathrm{E}=1 \mathrm{~V} / \mathrm{cm})$ for the two-electrode system and for the M, B1 and B2 protocols were approximately $73 \%, 83 \%$ and $85 \%$, respectively [12]. Therefore, for low energy, the shock outcome is significantly improved with the four-electrode system compared to the two-electrode system.

As the field strength increases to $3 \mathrm{~V} / \mathrm{cm}$ (see Fig. 4 ), the success rates for the $\mathrm{M}$ and $\mathrm{B} 1$ protocol reach a first plateau, while the success rate for the B2 protocol is already above the $95 \%$ level which results in the aforementioned low threshold $\mathrm{E}_{90}$. It is instructive to look at the mean values of the success rate for this shock strength $(E=3 \mathrm{~V} / \mathrm{cm}): 75 \%(\mathrm{M}), 77 \%$ (B1) and $97 \%$ (B2). For the same field value $(E=3 \mathrm{~V} / \mathrm{cm})$, the corresponding mean values for the two-electrode system were $44 \%(\mathrm{M})$, $44 \%$ (B1) and 46\% (B2). By comparing the observed defibrillation mechanisms at energy equals to $3 \mathrm{~V} / \mathrm{cm}$ in Fig. 4, we see that the main difference between the B2 protocol and the other two protocols is the very large percentage of delayed block defibrillation.

In order to investigate this result further, we have analyzed the delayed block mechanism in greater detail. We have proceeded in the following way: for every defibrillation trial that was successful via the delayed block mechanism we have kept the value of the front location where the front was last seen. This point on the ring corresponds to $\phi_{f}\left(t=t^{\prime}\right)$ (see Fig.1), where $t^{\prime}$ indicates the time where the front is blocked.

The distributions of the $\phi_{f}\left(t^{\prime}\right)$ points are shown in histograms in Fig.6. In Fig.6, the labels $X_{1}, X_{2}, X_{3}$ and $X_{4}$ refer to the position of the electrodes matching those in Fig.2. From Fig.6, one clearly sees that M protocol displays two higher peaks just before the two cathodes located at $X_{2}$ and $X_{4}$. Since all fronts used for building the histograms are propagating counterclockwise (i.e. $\phi_{f}$ increases with time), we conclude that the delayed block mechanism occurrs due to tissue depolarization by the cathode (the surviving front could not go through the refractory region created by a cathodal stimulus). The histogram (Fig.6, middle graph) corresponding to the B1 protocol shows two high peaks but in this case right before the electrodes located at $X_{1}$ and $X_{3}$. These electrodes have a second phase that is depolarizing (cathodal stimulus) and therefore generate also a refractory region as in the case of the protocol M. The results for the protocol B2 are somewhat different and more interesting. The histogram (Fig.6, lower graph) shows four peaks just before each of the four electrodes. In this latter case, all the electrodes have a cathodal character that generates a refractory region. Thus the high success rate of defibrillation for the B2 protocol for $E=3 \mathrm{~V} / \mathrm{cm}$ is explained as result of the combined properties of the $\mathrm{M}$ and $\mathrm{B} 1$ protocols where delayed block can occur behind each of the four electrodes.

In order to illustrate the discussion of the previous paragraph, we have constructed space-time plots corresponding to two different initial conditions (example A and example B) in Fig. 7. All the defibrillation protocols were tested with two different initial conditions. Both sets show examples with shock strengths corresponding to $E=1 \mathrm{~V} / \mathrm{cm}$ and $E=3 \mathrm{~V} / \mathrm{cm}$. In the cases displayed in Fig. 7, all the successful defibrillations were obtained via the delayed block mechanism. Let us first consider example A with a shock with $E=3 \mathrm{~V} / \mathrm{cm}$. Figures 7.(a4-a6) show an unsuccessful $\mathrm{M}$ and successful B1 and B2 shocks. The latter two succeded because of a front blockage at $\mathrm{X}_{3}$. The second phase for biphasic shocks is cathodal at location $\mathrm{X}_{3}$. However, protocols $\mathrm{M}$ are anodal at the position $\mathrm{X}_{3}$ and render the tissue more excitable and therefore hinder the front blockage. Figures 7.(b4-b6) show the results corresponding to the example $\mathrm{B}$. In this case, the protocols $\mathrm{M}$ and $\mathrm{B} 2$ result successful and the $\mathrm{B} 1$ protocol is unsuccessful. The front is blocked at location $\mathrm{X}_{4}$ for both the $\mathrm{M}$ and $\mathrm{B} 2$ shocks, where the tissue is refrac- 

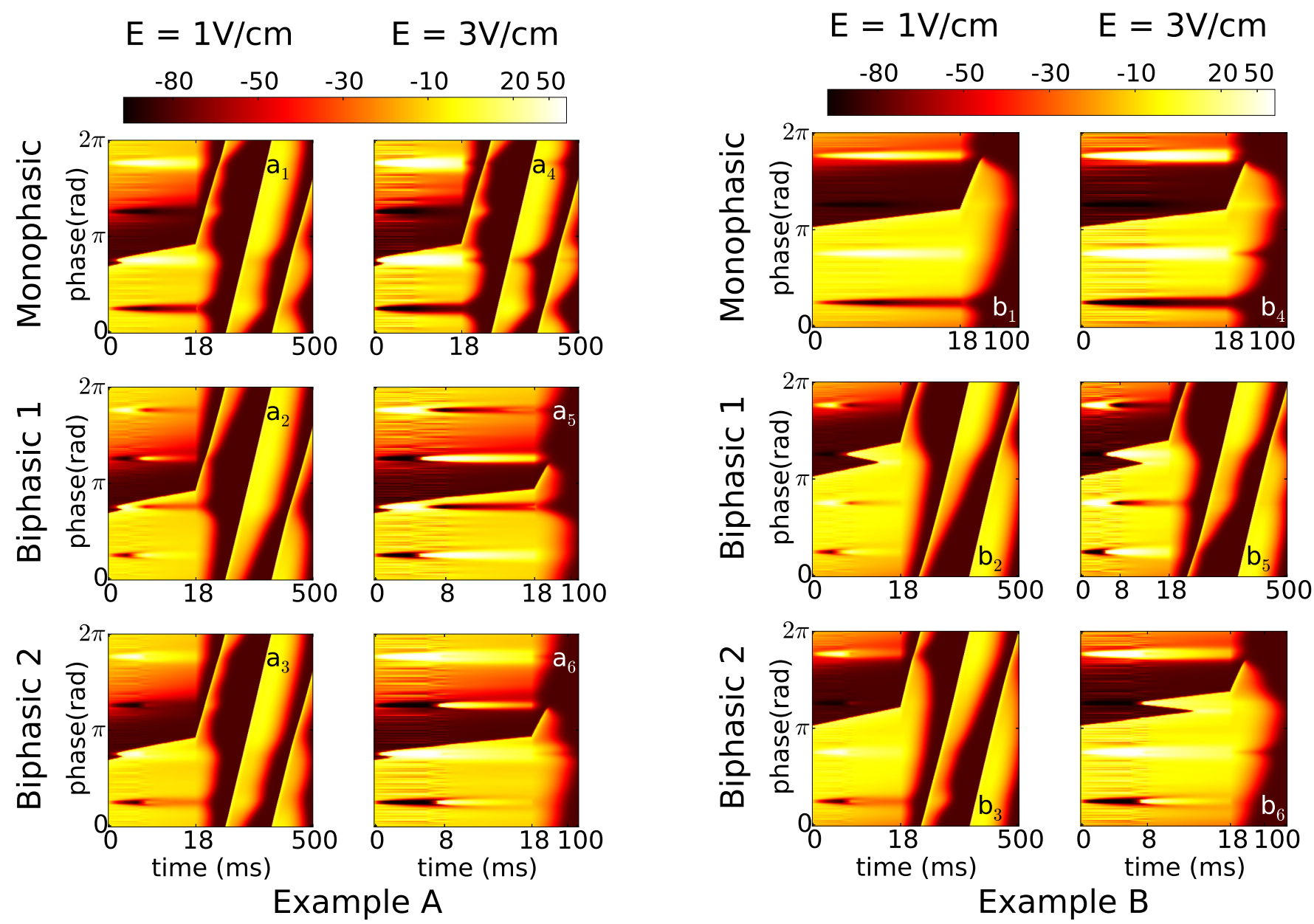

FIG. 7: Space-time plots of $\mathrm{V}_{m}$ for two different initial condition. Example A : $\phi_{f}(t=0)=2.22 \mathrm{rad}$ and Example B: $\phi_{f}(t=0)=3.27 \mathrm{rad}$. An external shock is applied during the first $8 \mathrm{~ms}$. To highlight the effect of the shock, the time resolution is one order of magnitude larger for $\mathrm{t}<18 \mathrm{~ms}$. Space-plots are shown for two shock strengths : $E=1 \mathrm{~V} / \mathrm{cm}$ and $E=3 \mathrm{~V} / \mathrm{cm}$ (columns) and all three protocols (rows). Note that we have used a nonlinear color scale for $\mathrm{V}_{m}$ to enhance clarity.

tory. Monophasic shocks at $\mathrm{X}_{4}$ are purely cathodal, but the second phases of $\mathrm{B} 1$ and $\mathrm{B} 2$ shocks at $\mathrm{X}_{4}$ are anodal. However, the duration of the second phase for B2 protocol is only $2 \mathrm{~ms}$ and therefore it is not long enough to render the tissue excitable. This is not the case for the protocol B1, for which the second phase lasts for $4 \mathrm{~ms}$ and it is long enough to hyperpolarize the tissue at $\mathrm{X}_{4}$ and allows the front to propagate through $\mathrm{X}_{4}$.

\section{Importance of the duration of the second phase}

Our analysis in the previous section demonstrates that the high success rate for the protocol $\mathrm{B} 2$ at $E=3 \mathrm{~V} / \mathrm{cm}$ is due to the appropriate durations of the first and the second phase of the shock. These durations are such that they produce the largest amount of refractory regions along the ring and therefore lead to the maximum elimination of the propagating fronts. Following this hy- pothesis it would be interesting to check the effect of a modification of the duration of the second phase of the protocol B2 while maintaining a fixed value of the field, i.e., $E=3 \mathrm{~V} / \mathrm{cm}$. In this section we have examined how the duration of the second phase affects the defibrillation success rate. Figure 8 displays the numerical results obtained for the success rate while varying the duration of the second phase. Let us first note that in Fig. 8 when the duration of the second phase is equal to $0 \mathrm{~ms}$ and $8 \mathrm{~ms}$ one retrieves a monophasic shock. The former case (i.e. 0 $\mathrm{ms}$ ) corresponds to the monophasic shock as schematized in Fig.2, while the latter case (i.e. $8 \mathrm{~ms}$ ) corresponds to a reversed placement of the anode and cathode with respect to the former case. The results of success rate for both monophasic shocks are comparable within the error bar of each other, which provides an additional check of the consistency of the simulations. In the same manner, when the duration of the second phase is equal to $4 \mathrm{~ms}$ one gets the protocol $\mathrm{B} 1$ results. 


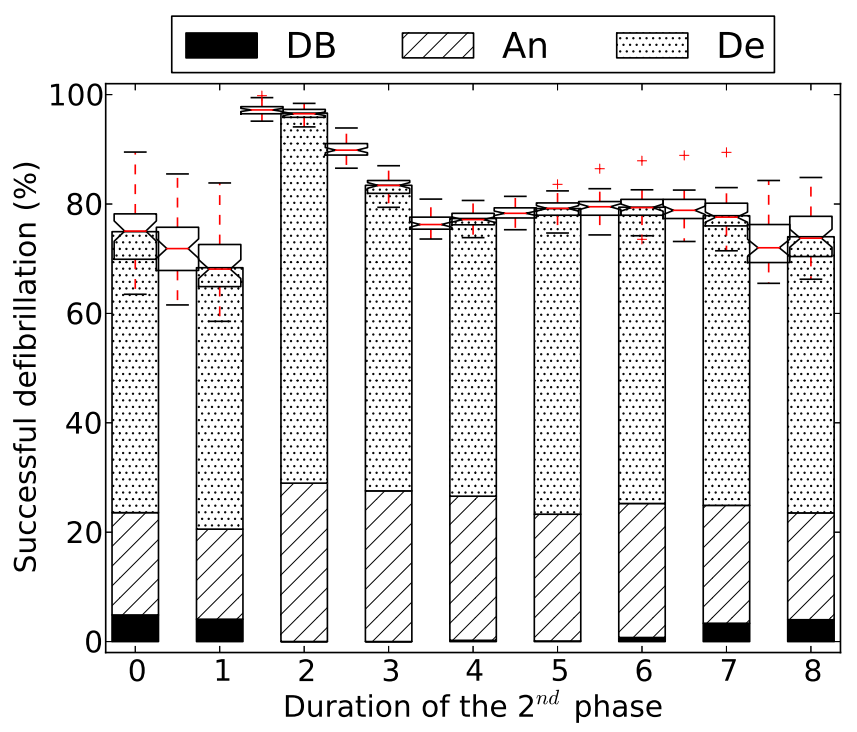

FIG. 8: Dependance of the defibrillation success rate on the duration of the second phase (in ms) for the biphasic protocols. The shock strength is fixed to $E=3 \mathrm{~V} / \mathrm{cm}$. The total shock duration is also kept fixed at $8 \mathrm{~ms}$.

The important message from Fig. 8 is that the percentage of defibrillation exhibits a marked maximum for a second phase duration in the range of $1.5 \mathrm{~ms}$ to $2.5 \mathrm{~ms}$ and a minimum for a second phase of duration around 1 ms. If we examine the defibrillation mechanisms corresponding to the shocks with a second phase duration of $2 \mathrm{~ms}$ with the other shocks, we see again that the main difference lies in the very high fraction of delayed block events. Indeed, when comparing the mechanisms for second phase duration equal to $2 \mathrm{~ms}$ and $3 \mathrm{~ms}$ for which the fractions of the annihilation are equal within the error bar, the difference is caused solely by the larger proportion of delayed block mechanism in the case of duration equals to $2 \mathrm{~ms}$.

\section{DISCUSSION SECTION}

\section{A. Discussion about the number of simulation runs}

In this paper we compare the relative efficacy of a twoand four-electrode system to eliminate the electrical activity present in a one-dimensional piece of cardiac tissue prior to the shock. In order to perform the comparison a very large number of simulations were carried out (4.8 millions simulations for the two-electrode [12] and 8.4 millions for the four-electrode system). An immediate comment comes into mind when such large number of simulations are performed. Was it necessary? Such large number of simulations yields very precise statistics and enables comparison and classification of the relative efficacy of the defibrillation protocols with high re-

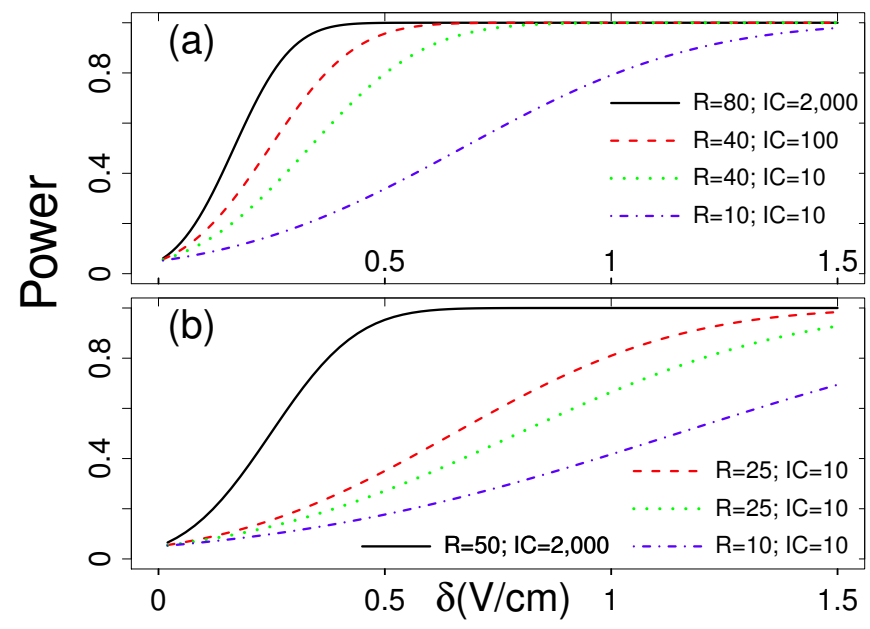

FIG. 9: (Color online) Statistical power calculation for the comparison between the $\mathrm{M}$ and $\mathrm{B} 2$ protocols (one-tail, $\alpha=0.05$ ). (a) two-electrode system (based on the GLM fitting). (b) four-electrode system (based on the GAM fitting). The parameters $R$ and $I C$ indicate the number of rings and initial conditions, respectively.

liability. One-dimensional simulations are not very time consuming and this large number of simulations can be handle in a few weeks with a dedicated small cluster of computers. When it comes to more realistic twoor three-dimensional simulations the simulation cost increases rapidly and therefore we can not hope doing such a large number of simulation runs. Here we study how the statistical power linked to the statistical comparison between different protocols would degrade when reducing the number of simulations. Let us recall that the power $(1-\beta)$ of a statistical test is related to the type-II error $\beta$ that is the probability of not identifying an effect from the data when there is one. In Fig. 9 we have computed the statistical power of the comparison between the $E_{90}$ of the monophasic and biphasic 2 protocols for the twoand four-electrode systems as a function of $\delta$ which is the true difference of the protocol efficacy assuming that the biphasic 2 is more efficient than the monophasic (one-tail testing, with significance level fixed to $\alpha=0.05$ ). In Fig. 9 , the parameters $R$ and $I C$ indicate the number of rings and initial conditions that were used for computing the power. The solid lines in Fig.9(a,b) correspond to the cases where all the simulation data were used for computing the power. If we recall that in the two-electrode system we have found that $E_{90}(\mathrm{~B} 2)-E_{90}(\mathrm{M}) \approx 1 \mathrm{~V} / \mathrm{cm}$ and if we assume that this value is close to $\delta$, we find by examining Fig. 9(a) that we have a statistical power very close to one if we use all the simulation data. Figure 9 (a) shows that for a fixed value of $\delta$, the power will decrease with $R$ and $I C$. We observe that with $R=10$ and $I C=10$, the power is about $80 \%$ for $\delta \approx 1 \mathrm{~V} / \mathrm{cm}$ which is a decent value for the statistical power. Figure $9(\mathrm{~b})$ examines the variation of the statistical power for the four-electrode system and we found that a large reduc- 
TABLE IV: Energy comparison for $90 \%$ success defibrillation for the two-electrode and four-electrode systems and all the protocols

\begin{tabular}{lc|c}
\hline \hline & $\begin{array}{c}2 \text { electrodes } \\
\text { Energy }(90 \%)\end{array}$ & $\begin{array}{c}4 \text { electrodes } \\
\text { Energy }(90 \%)\end{array}$ \\
\hline Monophasic & $200 \mathrm{~J}$ & $99 \mathrm{~J}$ \\
Biphasic 1 & $162 \mathrm{~J}$ & $119 \mathrm{~J}$ \\
Biphasic 2 & $148 \mathrm{~J}$ & $24 \mathrm{~J}$ \\
\hline \hline
\end{tabular}
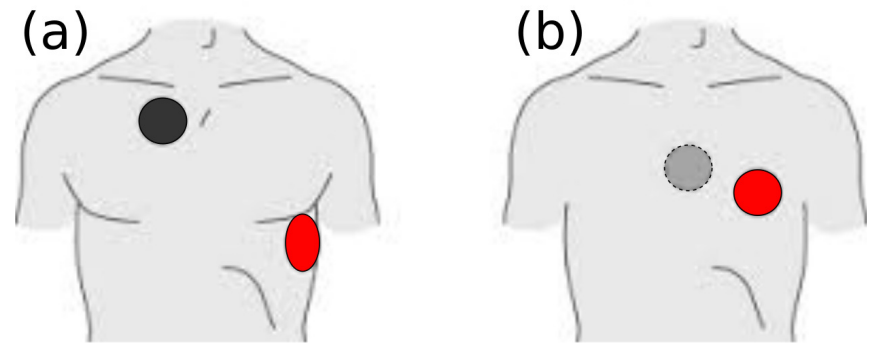

FIG. 10: (Color online). The two standard electrode positions for the two-electrode systems. (a)

Anterior-apex and (b) Anterior-posterior. These could be combined at once to offer a four-electrode system.

tion of simulation runs would still give a decent statistical power.

\section{B. Findings and limitations}

Our numerical simulations were analyzed in detail and the defibrillation events were classified into four mechanism [12], direct block, annihilation, delayed block and direct activation. We have found that for high energy shocks for which the direct activation is the prevailing mechanism, the two systems (two- and four-electrode devices) do not differ much in rate of success and underlaying defibrillation mechanisms. The prevailing mechanism behind the threshold $\mathrm{E}_{90}$ for the two-electrode system is the direct activation mechanism, for which the shock energy must be high enough to excite sufficient virtual electrodes in the cardiac tissue. In contrast, the threshold $\mathrm{E}_{90}$ for the biphasic 2 protocol in the case of the fourelectrode system is as low as $E=2.32 \mathrm{~V} / \mathrm{cm}$. For such low fields, the defibrillation is achieved only by front to front interactions or interactions of the front with refractory tissue. We have shown that the mechanism behind the superiority of the biphasic 2 protocol is the delayed block mechanism but that a subtle tuning of the timing of the second phase of the B2 protocol is necessary to obtain very high defibrillation performance. For $E=3 \mathrm{~V} / \mathrm{cm}$, we have found an optimum for a B2 protocol of $6 \mathrm{~ms}$ (phase 1) and $2 \mathrm{~ms}$ (phase2) durations.

The main results of the paper are summarized in Table IV listing the values of the energy associated with a
$90 \%$ probability of defibrillation for the two- and fourelectrode systems and for all the protocols studied here. We take as a reference the $200 \mathrm{~J}$ energy for the monophasic shock in the two-electrode system which is a meaningful value according to [4]. The relatively low value obtained for the biphasic 2 protocol with a four-electrode system is very striking and would mean a very efficient defibrillator device if these results are confirmed in more realistic simulations and experiments. One clear limitation of the study is that we have constrained the dynamics to a one-dimensional piece of cardiac tissue, thus eliminating many interesting 2D and 3D fibrillation dynamics such as spiral and scroll waves [43, 44].

Our study indicates that the timing of the shock and the duration of the second phase of the biphasic shock are crucial parameters in determining the efficacy of the shock. We believe that the electrode location is also an important parameter and that it should be studied more thoroughly, the assumption of equally spaced electrode made in this paper should be revisited (see Fig.2).

The discussion (see Sec.IV A) about the number of simulations needed to perform a reliable comparison between the different protocols indicates that a much smaller number of simulations could be used when dealing with $2 \mathrm{D}$ and $3 \mathrm{D}$ systems if the true differences between the efficacy of the protocols stay the same as in the onedimensional case. This will make it possible to do this study in the near future with the available computational capacity.

\section{Clinical realizations}

As far as we know, there have been only few attempts to test different multi-electrode configurations [45-47] but these suggest marked benefits in using a fourelectrode system.

Munsif et al. [46] in a clinical study compared dual, triple and quadruple electrode systems. A total of four different electrodes were used: two intravascular catheter electrodes, one active can shell electrode and one cutaneous patch electrode. A catheter electrode placed in the right ventricle was always cathodal for the first phase of the biphasic pulse, while other three possible electrodes were always anodal in the first phase of the biphasic pulse. The second catheter lead was positioned in the superior vena cava, the cutaneous patch electrode in the axillary position and the can electrode in the pectoral position. The lowest threshold was achieved with a combination of these four electrodes $((5.6 \pm 3.6) \mathrm{J})$, while the highest defibrillation threshold was achieved with the two-electrode system (right ventricle and superior vena cava, (14.2 \pm 6.4$) \mathrm{J})$.

Cooper et al. [47] performed another clinical investigation in which a total four electrodes were used to assess the atrial defibrillation threshold. One pair of electrodes was used to deliver a first shock, while a second pair of electrodes was used to deliver a subsequent shock. Elec- 
trodes were positioned in the right atrial appendage, the left subclavian vein, the proximal coronary sinus and distal coronary sinus. A significant reduction in defibrillation threshold was found for the two pair of electrodes delivering two subsequent pulses $((2.0 \pm 0.4) \mathrm{J})$ when compared with the standard pair of electrodes and one pulse $((5.1 \pm 1.8) \mathrm{J})$.

Implanted multi-electrode devices have also been patented [48-50]. For example, in a patent filed by Ideker et al. [50], the authors describe a similar approach to the one of Cooper et al. [47] where a total of four electrodes are used to deliver two sequential defibrillating pulses.

Yamanouchi et al. [51] tested a three-electrode internal defibrillator. They have shown that the addition of the extra electrode improves the defibrillation efficacy.

\section{CONCLUSIONS AND FUTURE WORKS}

In this paper we have studied defibrillation induced by three types of shock protocols, i.e., monophasic, biphasic 1 , and biphasic 2. The interest of the present study is that we have compared the efficacy of a four-electrode and a standard two-electrode system. The results of the defibrillation shocks were obtained through many numerical simulations on a one-dimensional ring of cardiac tissue. The initial state of the system, mimicking the arrhythmic state, is a reentrant wave that exhibits a discordant-alternans dynamics. All three four-electrode protocols are consistently more efficient than their corresponding two-electrode system counterpart. Among the three protocols studied, the biphasic protocol B2 is by far the most efficient. We have compared (see table IV) the energy associated to $90 \%$ defibrillation for the two and four-electrode systems. We have found that the protocols M, B1 and B2 in the four-electrode system save 50\%, 26\% and $84 \%$ of energy when compared to the two-electrode system. These important energy savings obtained with a four-electrode system open the door to some further studies that consider more realistic models of four-electrode defibrillators.

While our study only presents numerical results in a simplified geometry, it would be interesting to test our findings in a more detailed and realistic heart geometry. The possible gain obtained by a four-electrode system is further supported by the energy savings reported in the clinical studies in which a similar setup was used. Perhaps the easiest application can be taken in the hospital settings, where patients with the risk of ventricular fibrillation are prepared with four self-adhesive electrodes as shown in Fig. 10.

Given the advancement of the technology of the implanted defibrillators over the past thirty years and the optimistic results obtained with a four-electrode setup, one can hope that more efficient and less traumatic new defibrillators will be designed in the near future.

\section{ACKNOWLEDGMENTS}

The authors acknowledge the financial support offered by MICINN through Grant No. FIS2011-28820-C0202. A.Šimić also acknowledges the support of the "Asociación de Amigos" of University of Navarra through a PhD fellowship. DL acknowledges partial financial support from "Basal Program Center for Development of Nanoscience and Nanotechnology (CEDENNA) and UTA-project 8750" (Chile).

\section{Appendix A: Automatic classification of defibrillation mechanisms using Artificial Neural Networks}

In this paper, each energy was tested with 100,000 trials for the four-electrode system. This large number of trials renders a manual classification cumbersome. Therefore, we have used an automatic classification through the analysis of the numerical simulation by the neural network method [42]. Each numerical simulation is characterized by a number of characteristic parameters: initial wave front $\left(\phi_{i}\right)$ and wave back $\left(\phi_{f}\right)$ phase (Fig.1), wavefront width $\left(\phi_{i}-\phi_{f}\right)$, percentage of depolarized tissue for different threshold levels $(-60 \mathrm{mV},-30 \mathrm{mV}$, $0 \mathrm{mV})$, trajectory points of the phase front, etc. These parameters are gathered in a vector of 50 entries that characterizes one trial simulation. The next step is to associate the vector corresponding to a simulation to a defibrillation events (if it occurs). The first phase of the neural network method consists in training the neural network by manually associating a vector to a mechanism. Here we have used 400 defibrillation events for each protocol (M, B1 or B2), i.e. 1,200 samples for a particular shock energy. The set is then divided into a training set (960 trials) and validation set (240 samples). Two types of neural networks were used here for further reducing the classification errors. One corresponds to a ANN with two hidden layers of 14 neurons and the other with a single hidden layer of 20 neurons. To further increase the accuracy, a total of five different partitions of the initial set were created (using the well-known technique of cross-validation). Thus each classification was evaluated with total of ten different networks. This allows to compute the standard error associated with the classification procedure. This procedure was repeated at four energy levels corresponding to: $E=1 \mathrm{~V} / \mathrm{cm}, 3 \mathrm{~V} / \mathrm{cm}$, $5 \mathrm{~V} / \mathrm{cm}$, and $7 \mathrm{~V} / \mathrm{cm}$. The neural network analysis was performed using the MATLAB's Neural Network Toolbox $[52]$. 
[2] R. Koster, P. Dorian, F. Chapman, P. Schmitt, S. OGrady, and R. Walker, Am. Heart J. 147 (5), 1 (2004).

[3] J. P. Nolan, J. Soar, D. A. Zideman, D. Biarent, L. L. Bossaert, C. Deakin, R. W. Koster, J. Wyllie, and B. Bittiger, Resuscitation 81, 1219 (2010).

[4] L. J. Morrison, R. M. Henry, V. Ku, J. P. Nolan, P. Morley, and C. D. Deakin, Resuscitation 84, 1480 (2013).

[5] M. W. Kroll and C. D. Swerdlow, "Lessons for the clinical implant," in Cardiac Bioelectric Therapy., edited by I. Efimov, M. Kroll, and P. Tchou (Springer, 2009) pp. 459-492.

[6] G. Boriani, M. Biffi, P. Silvestri, C. Martignani, C. Valzania, I. Diemberger, C. Moulder, G. Mouchawar, M. Kroll, and A. Branzi, Heart Rhythm 2, 708 (2005).

[7] T. Tokano, D. Bach, J. Chang, J. Davis, J. J. Souza, A. Zivin, B. P. Knight, R. Goyal, K. C. Man, F. Morady, and S. A. Strickberger, J. Card. Electrophys. 9, 791 (1998).

[8] T. Schneider, P. Martens, H. Paschen, M. Kuisma, B. Wolcke, B. Gliner, J. Russell, W. Weaver, L. Bossaert, and D. Chamberlain, Circulation 102, 1780 (2000).

[9] P. Martens, J. Russell, B. Wolcke, H. Paschen, M. Kuisma, B. Gliner, W. Weaver, L. Bossaert, D. Chamberlain, and T. Schneider, Resuscitation 49, 233 (2001).

[10] C. Henriquez, Crit. Rev. Biomed. Eng. 21, 1 (1993).

[11] G. W. Beeler and H. Reuter, J. Physiol. 268, 177 (1977).

[12] J. Bragard, A. Simic, J. Elorza, R. O. Grigoriev, E. M. Cherry, R. F. Gilmour, N. F. Otani, and F. H. Fenton, Chaos 23, 3119 (2013).

[13] L. Glass and M. E. Josephson, Phys. Rev. Lett. 75, 2059 (1995).

[14] A. Pumir and V. I. Krinsky, Physica D 91, 205 (1996).

[15] T. Krogh-Madsen and D. J. Christini, Phys. Rev. E 80, 021924 (2009).

[16] S. Sinha and D. J. Christini, Phys. Rev. E 66, 061903 (2002).

[17] P. Comtois and A. Vinet, Chaos 12, 903 (2002).

[18] N. F. Otani, IEEE Trans. Biomed. Eng. 58, 2013 (2011).

[19] R. Plonsey, Biophys. J. 39, 309 (1982).

[20] V. G. Fast, S. Rohr, A. M. Gillis, and A. G. Kleber, Circulation Res. 82, 375 (1998).

[21] C. M. Ripplinger and I. R. Efimov, "The virtual electrode hypothesis of defibrillation," in Cardiac Bioelectric Therapy., edited by I. Efimov, M. Kroll, and P. Tchou (Springer, 2009) pp. 331-356.

[22] T. Desplantez, E. Dupont, N. J. Severs, and R. Weingart, J. Membrane Biol. 218, 13 (2007).

[23] M. Courtemanche, Chaos 6(4), 579 (1996).

[24] R. Guttman and L. Hachmeister, Biophys. J. 12, 552 (1972).

[25] B. A. Roth, IEEE Trans. Biomed. Eng. 42, 1174 (1995).

[26] E. Cerbai, M. Barbieri, and A. Mugelli, Circulation 94, 1674 (1996).

[27] H. Yu, F. Chang, and I. S. Cohen, J. Physiol. 485, 469 (1995).

[28] R. Ranjan, N. Chiamvimonvat, N. V. Thakor, G. F. Tomaselli, and E. Marban, Biophys. J. 74, 1850 (1998).

[29] X. Zhou, W. M. Smith, D. L. Rollins, and R. E. Ideker, Am. J. Physiol. 271, H2536 (1996).
[30] K. A. DeBruin and W. Krassowska, Ann. Biomed. Eng. 26, 584 (1998).

[31] J. M. Pastore, S. D. Girouard, K. R. Laurita, F. G. Akar, and D. S. Rosenbaum, Circulation 99, 1385 (1999).

[32] S. Balay, J. Brown, K. Buschelman, W. D. Gropp, D. Kaushik, M. G. Knepley, L. C. McInnes, B. F. Smith, and H. Zhang, "PETSc Web page," (2012), http://www.mcs.anl.gov/petsc.

[33] Y. Saad and M. H. Schultz, Siam J. Sc. \& Stat. Comp. 7, 856 (1986).

[34] D. McFadden, "Conditional logit analysis of qualitative choice behaviour," in Frontiers in Econometrics, edited by P. Zarembka (New York, Academic Press, 1974) pp. $105-142$.

[35] S. Wood, Generalized Additive Models: An Introduction with $R$ (Chapman and Hall/CRC, 2006).

[36] R Development Core Team, $R$ : A Language and Environment for Statistical Computing, R Foundation for Statistical Computing, Vienna, Austria (2008).

[37] S. N. Wood, J. R. Stat. Soc. (B) 62, 413 (2000).

[38] S. N. Wood, J. R. Stat. Soc. (B) 73, 3 (2011).

[39] A. Davison and D. Hinkley, Bootstrap Methods and Their Application (Cambridge University Press, 1997).

[40] P. S. Chen, N. Shibata, E. G. Dixon, R. O. Martin, and R. E. Ideker, Circulation 73, 1022 (1986).

[41] E. G. Dixon, A. S. Tang, P. D. Wolf, J. T. Meador, M. J. Fine, R. V. Calfee, and R. E. Ideker, Circulation 76, 1176 (1987).

[42] C. Bishop, Neural Networks for Pattern Recognition (Oxford University Press, 1995).

[43] J. Weiss, P.-S. Chen, Z. Qu, H. Karagueuzian, and A. Garfinkel, Circulation Res. 87 (12), 1103 (2000).

[44] E. Cherry, F. Fenton, and R. J. Gilmour, Am. Heart J. 302 (12), H2451 (2012).

[45] J. Rhude, J. Sweeney, J. Reighard, and T. Brandon, in Proceedings of the First Joint BMES/EMBS Conference. 1999 IEEE Engineering in Medicine and Biology 21st Annual Conference and the 1999 Annual Fall Meeting of the Biomedical Engineering Society (Cat. No.99CH37015), Vol. 1 (IEEE, 1999) p. 317.

[46] A. N. Munsif, S. Saksena, P. Degroot, R. B. Krol, P. Mathew, I. Giorgberidze, R. R. Kaushik, and R. Mehra, Am. J. Cardiol. 79, 1632 (1997).

[47] R. A. S. Cooper, V. J. Plumb, A. E. Epstein, G. N. Kay, and R. E. Ideker, Circulation 97, 2527 (1998).

[48] W. A. J. Tacker, C. F. Babbs, J. D. Bourland, and L. A. Geddes, "Apparatus for controlling cardiac ventricular tachyarrhythmias," European Patent No EP 0095726(A1) (1983).

[49] K. F. Smits, "Cardioversion and defibrillation lead method," United States Patent No US 4641656 (1987).

[50] R. E. Ideker, P. A. Guse, D. J. Lang, D. K. Swanson, and R. W. Dahl, "Low energy multiple shock defibrillation/cardioversion discharge technique and electrode configuration," United States Patent 5107834 (1992).

[51] Y. Yamanouchi, K. Mowrey, M. Niebauer, P. Tchou, and B. Wilkoff, Circulation 96 (12), 4400 (1997).

[52] MATLAB, version 7.12.0 (R2011a) (The MathWorks Inc., Natick, Massachusetts, 2011). 